\title{
Determinación de las propiedades físicas y químicas del material granular obtenido del barrido de las calles de bogotá, para usarlo como material de construcción
}

Determination of physical and chemical properties of granular material obtained from sweeping the streets in bogota for use as construction material

\section{Diego Garay Agudelo}

Arquitecto, magíster en Construcción. Docente de la Universidad Distrital Francisco José de Caldas. Bogotá, Colombia.

digarqmayor@yahoo.com

Clasificación del artículo: Investigación (conciencias)

Fecha de recepción: 21 de marzo de 2011

Fecha de aceptación: 29 de agosto de 2011

Palabras clave: Material de construcción, propiedades físicas, propiedades químicas, residuos sólidos urbanos.

Keywords: Construction materials, physical properties, chemical properties, municipal solid waste.

\section{RESUMEN}

En la actualidad existe una preocupación a nivel mundial por el desarrollo de materiales sostenibles y sustentables que contribuyan con la preservación y conservación de la vida, en este sentido las organizaciones internacionales vienen adelantando investigaciones sobre la adecuada disposición final de residuos sólidos que producen las ciudades. Como un pequeño aporte en la búsqueda de alternativas para la disposición final de esos residuos este artículo presenta las características físicas y químicas del material granular del barrido de las calles y avenidas de Bogotá, para usarlo como material de construcción. En la primera parte se analizan las propiedades físicas de acuerdo con las normas vigentes para este fin, y en la segunda parte se analizan las propiedades químicas.

\begin{abstract}
There is now a global concern for sustainable development and sustainable materials that contribute to the preservation and conservation of wildlife in this regard international organizations are advancing research on the proper disposal of municipal solid waste produce cities. As a small contribution in the search for alternatives for the disposal of such waste, in this article presents the physical and chemical characteristics of granular material of street sweeping avenues of Bogotá, to use as building material. In the first part discusses
\end{abstract}




\section{con-ciencias}

the physical properties in accordance with the rules for this purpose, and the second part analyzes the chemical properties.

\section{INTRODUCCIÓN}

El manejo actual de los residuos sólidos de Bogotá exige tomar decisiones que permitan optimizar la gestión, por tanto se desarrolla un Plan Estratégico que permita a la ciudad manejar los residuos sólidos en forma integral y eficiente, considerando las condiciones locales y socioeconómicas de su población, al igual que valorando varias iniciativas públicas y privadas para el manejo de los residuos; ofertas tecnológicas tanto locales como internacionales, así como una variedad de estudios sectoriales, que para su implementación requieren analizar a fondo los componentes de estos residuos.

La protección del medio ambiente, el cuidando, la conservación de recursos naturales, así como la disposición final de los residuos sólidos obtenidos del barrido de calles y avenidas, hacen más factible la práctica del reciclaje, si a esto se le agrega el ahorro del consumo energético que se pueda generar al optimizar el transporte de áridos naturales hacia los centros consumidores, el atractivo económico es un aliciente más para el reciclado de residuos sólidos.

La evaluación del material granular, desde un enfoque de desarrollo sostenible, requiere considerar aspectos socioeconómicos y medioambientales, estudiando el ciclo de vida, que contempla: su extracción, producción, distribución y utilización, los contaminantes que produce, lo cual permite comparar diversas estrategias y cuantificar los impactos negativos y sus beneficios, de tal forma que permita definir el proceso más sostenible para el desarrollo, utilización y reutilización de estos recursos.

En lo corrido del año 2009 las estadísticas de recolección de los residuos sólidos producto de la actividad de barrido son la no despreciable suma de $99.739,49$ toneladas [1]; los concesionarios prestadores de servicio público de aseo (LIME), reali- zan la actividad del barrido en la localidad a través de operarios "escobitas" con carros papeleros y escobas, ellos llevan a cabo el barrido a través de micro rutas distribuidas dentro del perímetro urbano de la localidad, estos residuos son embolsados y posteriormente transportados por los concesionarios al relleno sanitario Doña Juana, donde son dispuestos técnicamente.

La caracterización de los residuos sólidos del barrido de las calles y avenidas de Bogotá y en especial aquellos que podrían ser utilizados dentro de procesos constructivos con materiales granulares en altas cantidades, es un paso fundamental dentro del plan estratégico de manejo de residuos sólidos, ya que permiten determinar si los residuos provenientes de la superficie de la tierra por efectos de la erosión, ocasionada por la acción del viento, el agua, el desplazamiento de los vehículos, se pueden aprovechar como material de construcción.

La localidad de Usaquén tiene buena parte de su territorio protegida por suelo rural correspondiente a los cerros orientales, estos cerros proveen en un alto grado a sus calles y avenidas de sedimentos sólidos que periódicamente son barridos de forma manual o mecánica por empresas prestadoras de servicios públicos, que luego se depositan en botaderos.

La caracterización física y química del material de barrido de la localidad de Usaquén se realiza con base en los normas vigentes que establecen los requisitos y propiedades que deben cumplir los materiales de construcción, en este caso por tratarse de un material en forma de polvo la evaluación de las características físicas se hace teniendo en cuenta las normas para agregados pétreos para la preparación de mezclas de concreto y mortero, se realiza una evaluación comparativa con todos los parámetros técnicos, establecidos en la normatividad vigente, haciendo uso de ensayos estándar en la totalidad de las muestras recogidas, con el 
fin de garantizar certeza en los datos obtenidos de la investigación.

De cumplir con las especificaciones técnicas como material granular fino o como material llenante, esta investigación sirve como base a un sin número de proyectos, que no solo aportan en el desarrollo de materiales sostenibles para la actividad edificadora sino también en la protección y preservación del medio ambiente y el desarrollo social sostenible, lo cual le confiere importancia y trascendencia a esta investigación.

\section{ASPECTOS HISTÓRICOS}

Los primeros escritos que referencian el servicio de aseo en Bogotá, se remontan a la época de la Colonia durante la cual es descrito como una obligación del Estado, pero con graves problemas debido al descuido ciudadano en el manejo de los desechos sólidos.

En esa época la situación llegó a tal punto que el Virrey Amar y Borbón se quejaba por "el tiempo que debía emplear en solucionar el problema de los escombros y basuras que interrumpían el tráfico, afeaban a la ciudad y amenazaban la salud pública".

Con anterioridad su antecesor señalaba "en Santa Fe hay cuatro agentes de policía encargados de mantener limpias las calles: la lluvia, los gallinazos, los burros y los cerdos" [2].

El problema de las basuras es marcado y en 1808 el Virrey encarga a las reclusas de la Cárcel de Mujeres o del Divorcio para recoger las basuras que se acumulaban en los sitios públicos. Como el problema continuaba, en 1822 las autoridades designan a un vecino por cuadra para vigilar a los habitantes del sector y evitar que las basuras se abandonen en la vía pública; pero el comportamiento ciudadano impidió el éxito del programa y un texto de la época describe "las acequias se convierten en propagadores permanentes de suciedad".

Solo hasta 1884 se inició la organización del servicio de aseo a través de particulares. Para 1901, la recolección se realiza con 50 carros particulares, pero con cargo al erario público. Finalmente, en 1902 el servicio de aseo fue asumido por el municipio de Santa Fe de Bogotá que compra la maquinaria y lleva a cabo la contratación de personal para prestar el servicio de aseo en la ciudad.

Durante años la recolección de basuras es atendida directamente por la Alcaldía de Bogotá a través de una sección denominada Ramo de Aseo. En 1958 el Concejo Municipal crea la Empresa Distrital de Aseo e incrementa el número de obreros y maquinaria que permite el cubrimiento total de la demanda del servicio.

En 1960 se le modifica el nombre por el de Empresa Distrital de Servicios Públicos, EDIS. En 1989 se privatiza el $40 \%$ de la recolección de desechos sólidos y barrido de calles y en 1991 se privatiza otro $20 \%$ adicional.

Durante 36 años la EDIS tuvo la responsabilidad de la recolección, barrido de calles y disposición final de los desechos sólidos producidos por los habitantes de Bogotá; pero sus continuas crisis financieras y operativas mantuvieron a la ciudad en permanente emergencia sanitaria, es por ello que en 1993 el Concejo capitalino expide el Acuerdo 41, mediante el cual se ordena la liquidación de la citada empresa.

\subsection{La modernización}

En 1994 se expide la Ley 142 "Ley de Servicios Públicos", que permite al Estado entregar, en concesión, a la empresa privada la prestación de los servicios públicos, siempre bajo su control y supervisión.

Acogiendo lo ordenado por el Concejo Capitalino y bajo los parámetros de la Ley 142 de 1994, la Administración Distrital después de un proceso licitatorio entrega en concesión el servicio de recolección, barrido y limpieza de áreas públicas y la Disposición Final de Basuras. Para su supervisión, control y planeación el Alcalde Mayor crea en 1994, la Unidad Ejecutiva de Servicios Públicos - UESP, mediante Decreto 782.

Gracias a estas políticas la ciudad hoy cuenta con un organizado servicio que permite que en todas 


\section{con-ciencias}

las zonas de la capital se realice la recolección de basuras domiciliarias tres veces a la semana en horarios predeterminados, que se efectúa a través de contratos de concesión, con los consorcios Ciudad Limpia, Lime, Aseo Capital y Atesa.

Cada consorcio tiene a su cargo unas zonas determinadas y además de la recolección tiene a su cargo el barrido de vías principales y avenidas, poda de césped y corte de árboles.

Paralelo a este servicio también se realiza la recolección de residuos sólidos a los pequeños, medianos y grandes productores de basuras, pertenecientes básicamente a los establecimientos industriales y comerciales de la ciudad.

De igual forma, existe la ruta hospitalaria manejada por el consorcio Ecocapital Internacional que recoge los desechos hospitalarios y similares generados por las centros de docencia e investigación, bioterios y laboratorios de biotecnología, cementerios, morgues, funerarias y hornos crematorios, consultorios, clínicas, farmacias, centros de pigmentación y tatuajes, laboratorios veterinarios, centros de zoonosis, zoológicos, laboratorios farmacéuticos y de producción de dispositivos médicos, peluquerías y centros de estética. Tratados especialmente y luego dispuesto en la celda de patógenos.

El mejoramiento continuo del servicio de aseo ha sido una de las preocupaciones de la Administración Distrital, y Bogotá cuenta hoy con el Plan Maestro para el Manejo Integral de Residuos Sólidos, que regulariza el servicio para la optimización de los recursos y su tecnificación, para mejorar el servicio de aseo con planes a corto, mediano y largo plazo.

\subsection{Contexto general del área de estudio}

El área de estudio se localiza en la localidad uno Usaquén de la ciudad de Bogotá, la localidad de Usaquén está en el extremo nororiental de Bogotá. Limita al occidente con la Autopista Norte, que la separa de la localidad de Suba; al sur con la calle 100, que la separa de la localidad Chapinero; al norte con los municipios de Chía y Sopo; y al oriente con el municipio de La Calera.
El territorio de Usaquén lo configura una zona baja o plana, suavemente ondulada, y la zona montañosa está compuesta por formaciones sedimentarias de rocas arenosas, duras y resistentes a la erosión y por rocas arcillosas blandas, en las zonas más escarpadas y pendientes $\left(30^{\circ}-75^{\circ}\right)$ predominan los suelos de la serie Monserrate, derivada de areniscas bastante superficiales, de fertilidad baja y ácidos. De los cerros orientales bajan numerosas escorrentías y quebradas que marcaron los linderos de las grandes fincas y conforman hoy canales de aguas lluvias, las principales quebradas Santa Ana, El Contador, San Cristóbal, la Floresta y Torca [3].

Buena parte de su territorio corresponde a áreas protegidas en suelo rural correspondiente a los cerros orientales, más de la mitad es suelo urbano y existe una pequeña porción que está entre las áreas de expansión. En total abarca $6.531 .32 \mathrm{Ha}$ distribuidas así:

- Suelo urbano: 3.521.66 ha, que corresponden al $54 \%$ de la localidad.

- Zona de expansión urbana: 289.74 ha, el 4\% de la localidad.

- Zona rural y áreas protegidas: $2.719 .22 \mathrm{ha}$, el $42 \%$.

La zona de ladera de la localidad de Usaquén, que corresponde aproximadamente al $60 \%$ del área de la localidad, cuenta con 1.329 hectáreas calificadas en amenaza media por Fenómeno de Remoción en Masa.

\subsection{Barrido de calles}

El barrido de calles es un servicio importante en áreas urbanas. La cantidad de basura y polución que es arrojada a las calles por carros y peatones debe ser removida, no sólo para mejorar el aspecto de las vías y evitar el bloqueo de los desagües, sino también para reducir la polución que es lanzada a la atmósfera o que llega a los ríos y océanos a través de los desagües [4].

En el año 2009 hasta el mes de septiembre las estadísticas de recolección de los residuos producto de la actividad de barrido son de 99.739,49 toneladas distribuidas mensualmente de la siguiente manera: 
Tabla 1. Recolección Barrido de las Calles año 2009.

\begin{tabular}{|c|c|}
\hline Mes & Toneladas \\
\hline Enero & $10.997,27$ \\
\hline Febrero & $10.706,13$ \\
\hline Marzo & $11.593,46$ \\
\hline Abril & $11.694,55$ \\
\hline Mayo & $10.768,17$ \\
\hline Junio & $11.314,81$ \\
\hline Julio & $11.218,63$ \\
\hline Agosto & $10.488,59$ \\
\hline Septiembre & $10.957,88$ \\
\hline
\end{tabular}

Fuente. Unidad Administrativa Especial de Servicios Públicos, Radicado No. 2009EE-009128 de 29 de octubre de 2009.

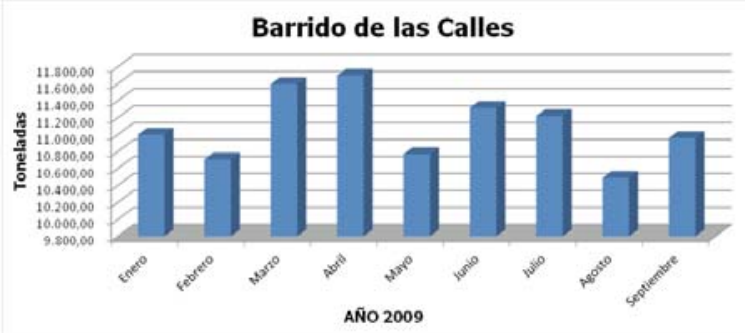

Fig. 1. Recolección barrido de las calles año 2009.

Fuente. Unidad Administrativa Especial de Servicios Públicos, Radicado No. 2009EE- 009128 de octubre de 2009 [5].

Lime S.A. E.S.P. es la empresa encargada de realizar los barridos manuales y mecánicos de la localidad de Usaquén, de los datos del último informe de gestión encontramos:

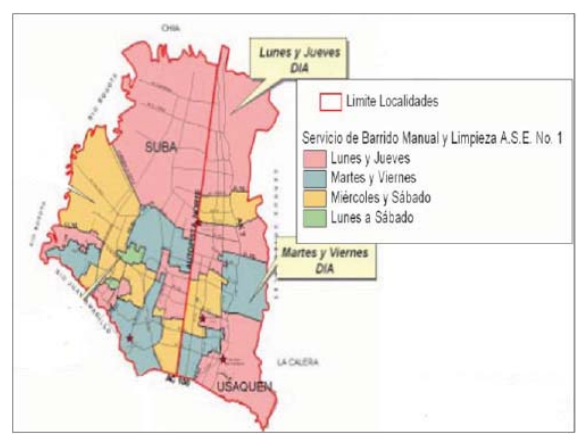

Fig. 2. Frecuencias y horarios de barrido en la localidad de Usaquén.

Fuente. LIME S.A. E.S.P. Informe de Gestión 2009
LIME barre manualmente $264.000 \mathrm{~km} / \mathrm{año} \mathrm{y}$ mecánicamente 54.000 km/año.

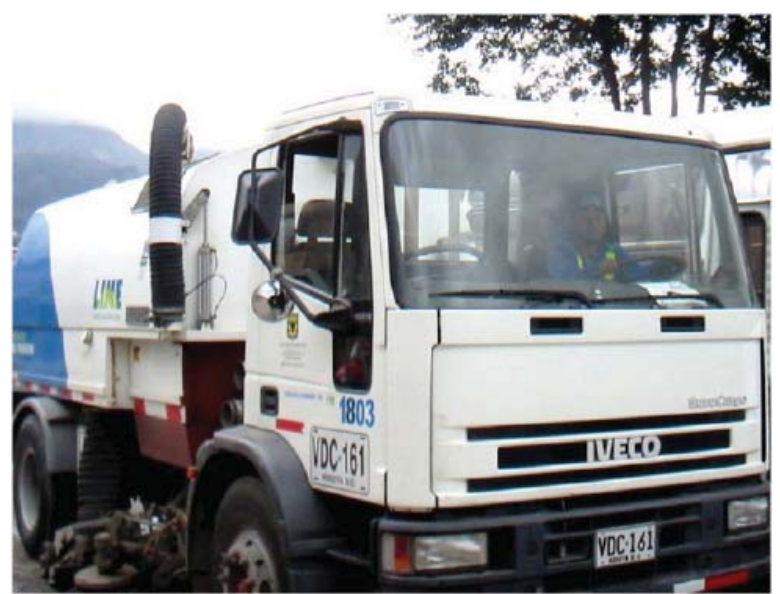

Fig. 3. Vehículo de barrido mecánico.

En cuanto al levantamiento de barrido LIME realiza 4.004 viajes al año y recoge la no despreciable suma de 17.090 ton/año.

Adicionalmente esta localidad tiene un cubrimiento del $100 \%$ de la totalidad de recolección de basuras, es una de las localidades con cubrimiento total.

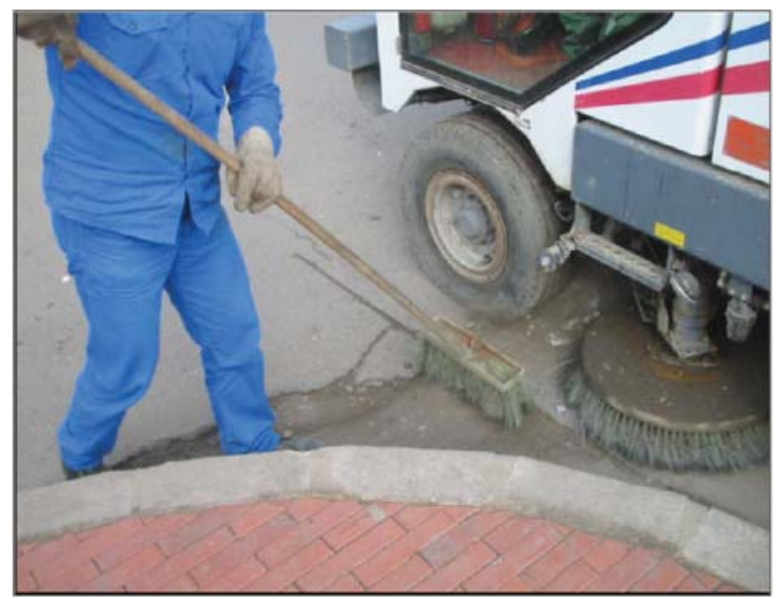

Fig. 4. Barrido manual y mecánico. 


\section{con-ciencias}

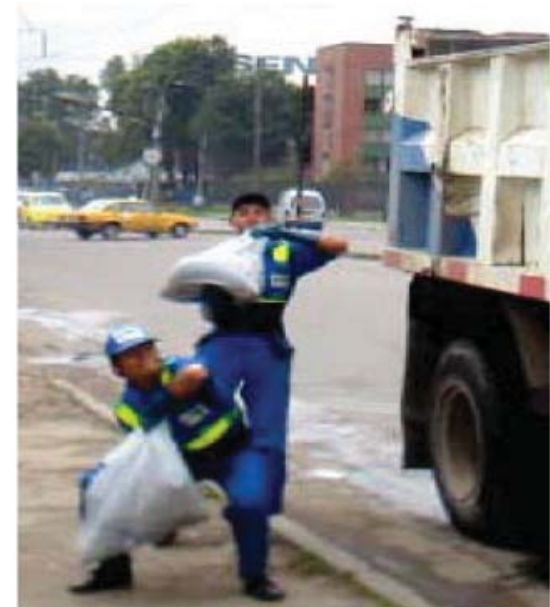

Fig. 5. Levantamiento de barrido en la localidad de Usaquén.

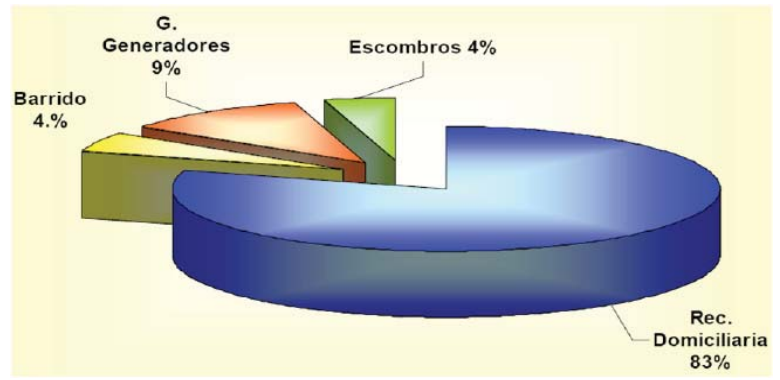

Fig. 6. Participación de residuos por servicio en la localidad de Usaquén.

Fuente: ob.cit.

\section{METODOLOGÍA}

\subsection{Análisis físico}

Para realizar el análisis de las muestras se sigue el procedimiento descrito en el "análisis por cuarteo", las muestras de material son tomadas sin-situ por los investigadores en un sector identificado de Bogotá y en diferentes puntos en la búsqueda de la formación de una muestra de laboratorio consistente [6].

Del análisis granulométrico se obtiene la distribución por tamaño de las partículas presentes en la muestra recogida, producto del barrido de las calles, esto se hace con el fin de realizar la clasificación mediante el sistema SUCS, este ensayo permite evaluar el tamaño de las partículas del material para luego determinar su composición química [7].

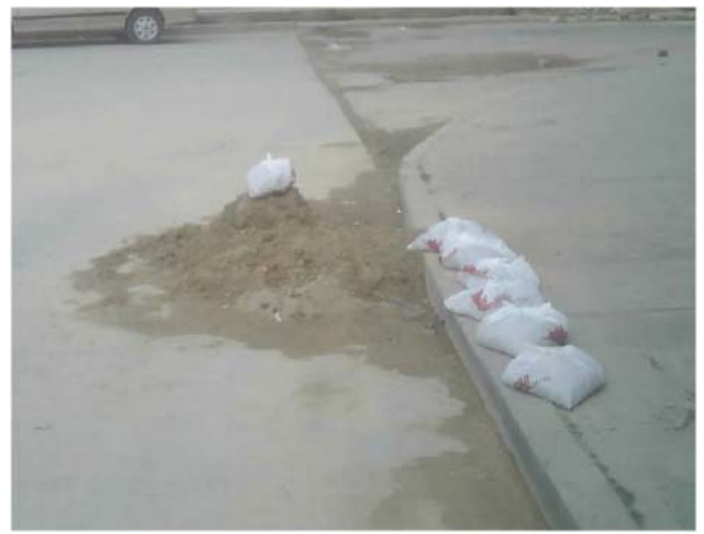

Fig. 7. Recolección de muestras

Para determinar y analizar la distribución de los tamaños se utiliza una serie de tamices normalizados y numerados tal como se muestra en la Fig. 7, dispuestos en orden descendente y basado en los parámetros de la norma NTC 77.

Para realizar la clasificación y evaluación del tamaño de las partículas de las muestras se toma como guía la clasificación del sistema SUCS [8].

Sin profundizar demasiado en el tema, el sistema SUCS divide los suelos primero en dos grandes grupos, de granos gruesos y de granos finos. Los primeros tienen más del 50 por ciento en peso de granos mayores que $0,08 \mathrm{~mm}$; se representan por el símbolo G, si más de la mitad en peso de las partículas gruesas son retenidas en tamiz $5 \mathrm{~mm}$, y por el símbolo $\mathbf{S}$, si más de la mitad pasa por tamiz $5 \mathrm{~mm}$.

A la $\mathbf{G}$ o a la $\mathbf{S}$ se les agrega una segunda letra que describe la graduación: $\mathbf{W}$, buena graduación con poco o ningún fino; $\mathbf{P}$, graduación pobre, uniforme o discontinua con poco o ningún fino; $\mathbf{M}$, que contiene limo o limo y arena; $\mathbf{C}$, que contiene arcilla $o$ arena y arcilla.

Los suelos finos, con más del 50 por ciento bajo tamiz $0,08 \mathrm{~mm}$, se dividen en tres grupos, las arcillas (C), los limos (M) y limos o arcillas orgánicos (O). 
Estos símbolos están seguidos por una segunda letra que depende de la magnitud del límite líquido e indica la compresibilidad relativa: L, si el límite líquido es menor a $50 \mathrm{y} \mathrm{H}$ si es mayor.

Se considera que una buena granulometría es aquella que está constituida por partículas de todos los tamaños, de tal manera que los vacíos dejados por las de mayor tamaño sean ocupados por otras de menor tamaño y así sucesivamente [9].

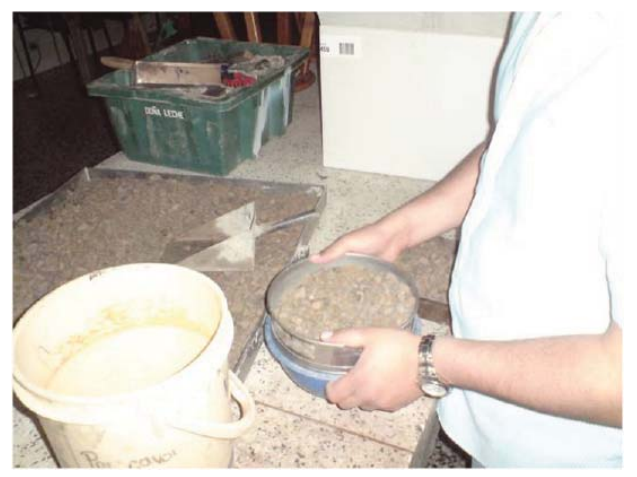

Fig. 8. Tamizado del material de barrido.

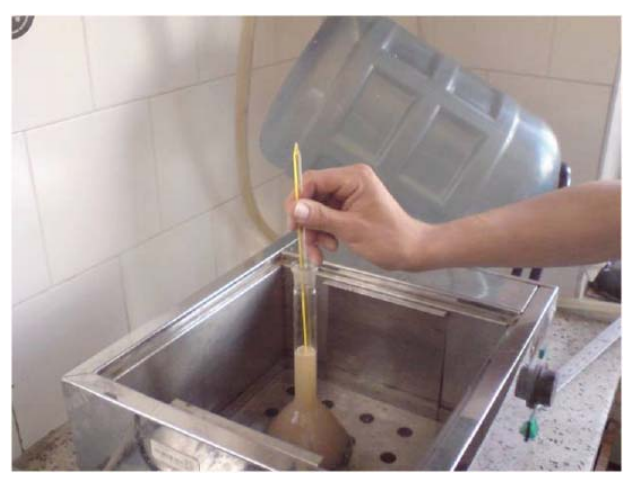

Fig. 9. Uso del picnómetro para estimación de pesos específicos.

El agregado fino consiste en arena natural que cumple con la Norma ICONTEC 174, actualiza- da. Para efectos de interpretación de la prueba de sanidad del agregado, se establece que en cinco ciclos del ensayo con sulfato de sodio, ejecutado según la Norma ICONTEC 126, actualizada, el agregado fino no deberá tener una pérdida en peso mayor del 6\%.

La granulometría de la arena natural, según los análisis de tamices, deberá conformarse con los requisitos de la Norma ICONTEC 174, actualizada. De acuerdo con esta norma, el módulo de finura de la arena deberá estar entre 2.2 y 3.4 [10].

\subsection{Análisis químico}

La toma de esta muestra se establece de igual forma que se realiza la muestra para el análisis físico, por el método de "análisis por cuarteo". Esta muestra se somete a un estudio mineralógico realizado por el Instituto Colombiano de Geología y Minería - INGEOMINAS; resultados relacionados en la Tabla 5.

Teniendo en cuenta la procedencia, la cual es de una roca sedimentaria identificada por el entorno y el lugar de estudio, su composición química de acuerdo con los resultados obtenidos del análisis mineralógico con un porcentaje de $91.03 \%$ correspondiente a Silicio expresado en un compuesto resultante de la combinación del sílice con el oxígeno(SiO2), que según la composición química de las arenas es el compuesto de mayor participación en estos materiales [11].

La arena sílice es un compuesto resultante de la combinación del sílice con el oxígeno. Su composición química $(\mathrm{SiO} 2)$ está formada por un átomo de sílice y dos átomos de oxígeno, formando una molécula muy estable que es insoluble en agua $y$ que en la naturaleza se encuentra en forma de cuarzo [12]. 


\section{RESULTADOS}

\subsection{Resultados del análisis físico}

Los resultados del análisis físico se encuentran en la Tabla 2.

Tabla 2. Resultados de ensayos de la caracterización física.

\begin{tabular}{|c|c|c|c|c|c|c|c|c|c|c|}
\hline \multirow{2}{*}{\multicolumn{2}{|c|}{$\begin{array}{l}\text { LABORATORIOS } \\
\text { REALIZADOS }\end{array}$}} & \multicolumn{7}{|c|}{ MUESTRA DE ENSAYO } & \multirow{2}{*}{ PROMEDIO } & \multirow{2}{*}{ NORMA } \\
\hline & & 1 & 2 & 3 & 4 & 5 & 6 & 7 & & \\
\hline \multicolumn{2}{|c|}{$\begin{array}{l}\text { Porcentaje de material fino que } \\
\text { pasa el tamiz No. } 200 \text { en los } \\
\text { agregados }(\%)\end{array}$} & 7,00 & 8,02 & 9,52 & 6,32 & 9,25 & 10,11 & 7,56 & 8,25 & $\begin{array}{l}\text { INV E-214-07 } \\
\text { NTC } 78\end{array}$ \\
\hline \multicolumn{2}{|c|}{ Clasificación SUCS } & SW-SM & SW-SM & SW-SM & SW-SM & SW-SM & SW-SM & SW-SM & N.A. & ASTMD 2487 \\
\hline \multicolumn{2}{|c|}{ Materia Organica } & 3,65 & 3,80 & 3,90 & 3,10 & 4,30 & 3,50 & 3,35 & 3,66 & $\begin{array}{c}\text { INV E - } 121 \\
-3\end{array}$ \\
\hline \multicolumn{2}{|c|}{ Módulo de Finura } & 2,21 & 2,52 & 2,35 & 2,85 & 2,65 & 2,7 & 3,1 & 2,63 & $\begin{array}{c}\text { INV E-213-07 } \\
\text { NTC } 77\end{array}$ \\
\hline \multicolumn{2}{|c|}{$\begin{array}{l}\text { Contenido aproximado de } \\
\text { materia organica. Metodo de } \\
\text { quemado. }\end{array}$} & 2,44 & 2,73 & 3,31 & 2,85 & 3,52 & 2,84 & 3,11 & 2,97 & INV E-121-07 \\
\hline \multicolumn{2}{|c|}{ Humedad Natural (\%) } & 3,35 & 3,86 & 4,12 & 3,21 & 3,35 & 4,02 & 3,35 & 3,61 & INV E-121-07 \\
\hline \multirow{3}{*}{$\begin{array}{l}\text { Gravedad } \\
\text { especifica } \\
(\mathrm{g} / \mathrm{cm} 3)\end{array}$} & $\begin{array}{l}\text { Gravedad } \\
\text { Esp.apa. } \\
\text { 20/20 }\end{array}$ & 2,575 & 2,567 & 2,652 & 2,568 & 2,576 & 2,568 & 2,567 & 2,58 & $\begin{array}{c}\text { INV E-222-07 } \\
\text { INV E-223-07 } \\
\text { NTC } 237 \text { NTC } \\
176\end{array}$ \\
\hline & $\begin{array}{l}\text { Gravedad } \\
\text { Esp. S.S.S } \\
\text { 20/20 C }\end{array}$ & 2,616 & 2,612 & 2,654 & 2,614 & 2,618 & 2,598 & 2,612 & 2,62 & $\begin{array}{c}\text { INV E-222-07 } \\
\text { INV E-223-07 } \\
\text { NTC } 237 \text { NTC } \\
176\end{array}$ \\
\hline & $\begin{array}{l}\text { Gravedad } \\
\text { Nominal } \\
20 / 20 \text { C }\end{array}$ & 2,687 & 2,689 & 2,695 & 2,688 & 2,685 & 2,630 & 2,690 & 2,68 & $\begin{array}{l}\text { INV E-222-07 } \\
\text { INV E-223-07 } \\
\text { NTC } 237 \text { NTC } \\
176\end{array}$ \\
\hline \multicolumn{2}{|c|}{ Porcentaje de absorción (\%) } & 1,63 & 1,77 & 1,85 & 1,69 & 1,71 & 1,64 & 1,72 & 1,72 & $\begin{array}{c}\text { INV E-222-07 } \\
\text { INV E-223-07 } \\
\text { NTC } 237 \text { NTC } \\
176\end{array}$ \\
\hline \multirow{2}{*}{$\begin{array}{l}\text { Masas Unitar- } \\
\text { ias }(\mathrm{g} / \mathrm{cm} 3)\end{array}$} & Suelta & 1,400 & 1,200 & 1,400 & 1,200 & 1,400 & 1,400 & 1,400 & 1,343 & $\begin{array}{l}\text { INV E-217-07 } \\
\text { NTC } 92\end{array}$ \\
\hline & Compacta & 1,5 & 1,3 & 1,100 & 1,1 & 1,3 & 1,2 & 1,2 & 1,24 & $\begin{array}{l}\text { INV E-217-07 } \\
\text { NTC } 92\end{array}$ \\
\hline \multirow{3}{*}{$\begin{array}{l}\text { Limites } \\
\text { de consistencia }\end{array}$} & $\begin{array}{c}\text { Limite } \\
\text { Liquido (\%) }\end{array}$ & NL & NL & NL & NL & NL & NL & NL & N.A. & \multirow{3}{*}{$\begin{array}{c}\text { INV E-126 } \\
-07\end{array}$} \\
\hline & $\begin{array}{c}\text { Limite } \\
\text { Platico (\%) }\end{array}$ & NP & NP & NP & NP & NP & NP & NP & N.A. & \\
\hline & $\begin{array}{l}\text { Indice de } \\
\text { plasticidad } \\
(\%)\end{array}$ & 0 & 0 & 0 & 0 & 0 & 0 & 0 & 0,00 & \\
\hline
\end{tabular}




\section{con-ciencias}

\subsection{Comparación de las curvas granulométricas de las siete (7) muestras}

La Fig. 10 muestra las diferentes curvas granulométricas

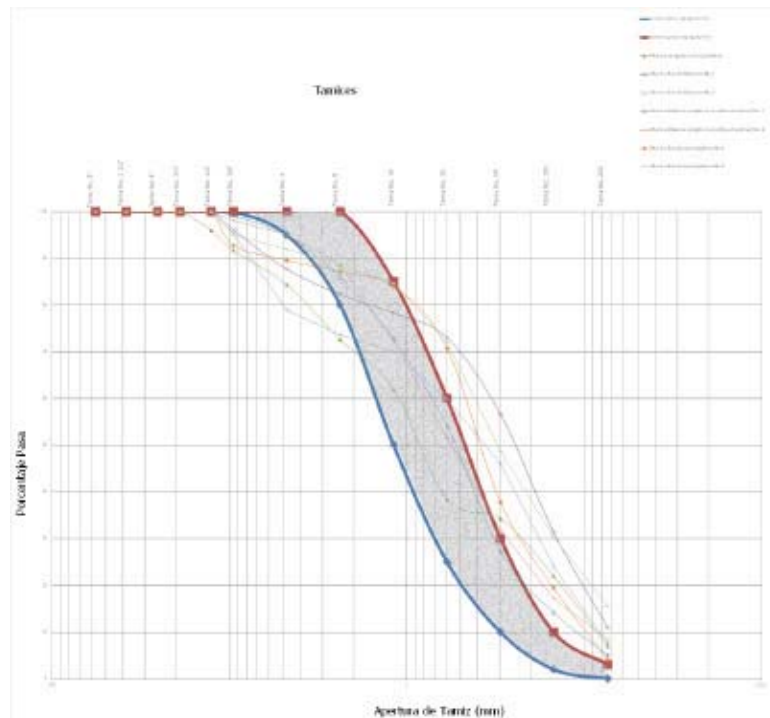

Fig. 10. Curvas granulométricas del material de barrido.

\subsection{Comparación de la Caracterización física con las especificaciones técnicas del material obtenido para mezclas de concreto}

En la Tabla 3 se encuentra la franja granulométrica permitida para las arenas según la NTC-174

Tabla 3. Franja granulométrica permitida para las arenas según la NTC-174.

\begin{tabular}{|c|c|c|c|c|c|c|c|c|}
\hline REFERENCIA & $3 / 8 "$ & No. 4 & No. 8 & No. 16 & No. 30 & No. 50 & No. 100 & No. 200 \\
\hline $\begin{array}{c}\text { Arena Norma } \\
\text { NTC-174 }\end{array}$ & $100-100$ & $100-95$ & $100-80$ & $85-50$ & $60-25$ & $30-10$ & $10-2$ & $7-0$ \\
\hline
\end{tabular}

Para lograr una caracterización precisa y estimar los posibles usos del material derivado, como material de construcción, se hace una comparación con los parámetros promedios de los resultados obtenidos en el laboratorio.

La NTC-174 nos brinda unos rangos que deben tener las arenas, para cumplir con las gradaciones correspondientes para permitirnos hacer mezclas de concreto de excelentes especificaciones técnicas, y esta investigación permite establecer que la franja granulométrica obtenida individualmente y en promedio, no cumple con este parámetro y debería ser rechazada como un uso potencial del material para ese propósito.

Este análisis se permite hacer de manera individual comparando los rangos de la NTC-174 establecidos en la Tabla 3, con el porcentaje establecido por los valores críticos de Chauvenet para muestra 1 obtenida en la carrera séptima con calle 134 (intersección costado Sur-Norte.)

Una muestra analizada para llevar a cabo la caracterización física del material que pasa el tamiz No. 200 por medio del ensayo NTC 78, el material que pasa el tamiz No. 200 está representado por limo, arcilla y materia orgánica, este a su vez es perjudicial para preparar mezclas de concreto y en la NTC-174 se acepta hasta un siete por ciento de este material $(7 \%)$ y algunas entidades exigentes permiten en sus especificaciones técnicas mezclas de concreto con un máximo de cinco por ciento 


\section{con-ciencias}

(5\%), considerando que si existe menos del 20 por ciento la mezcla tiene una calidad superior.

Al realizar el cálculo del módulo de finura se obtuvo un resultado individual por debajo del rango mínimo permitido y solo una muestra obtenida cumplió con los parámetros de la NTC -174, sin embargo, en promedio el material obtenido del barrido tuvo un 2.63 de MF. Esto nos indica que contamos con una arena que no se encuentra entre los intervalos especificados que son 2,7 y 3,1 ; de esta manera permite decir que es una arena no adecuada para diseñar una buena mezcla para concreto. La absorción es quizás la propiedad del agregado que más influye en la consistencia del concreto, puesto que las partículas absorben agua directamente en la mezcladora, disminuyendo la manejabilidad de la mezcla, por este motivo es importante llevar a cabo este ensayo que arrojó en promedio una absorción de 1.72 , que a criterio de los investigadores es normal y no afecta la consistencia del concreto o del mortero.

\subsection{Aplicación del criterio excluyente de Chauvenet para los datos obtenidos de la caracterización física}

En las Tabla 4, 5, 6, 7 y 8 se encuentran los resultados de la Aplicación del criterio excluyente de Chauvenet para los datos obtenidos de la caracterización física.

Tabla 4. Porcentaje de finos que pasan el Tamiz No. 200.

\begin{tabular}{|c|c|}
\hline Media aritmética & $\mathrm{X}=8.25$ \\
\hline Desviación estándar & $\mathrm{S}=1.41$ \\
\hline Valor crítico de Chauvenet & $1.80($ PARA N=7) \\
\hline Smáx1 & $16.32-8.251=1.93$ \\
\hline Smáx1 /S & $\begin{array}{c}1.93 / 1.41=1.37<1.80 \\
\text { El dato SI es pertinente }\end{array}$ \\
\hline Smáx2 & $110.11-8.251=1.86$ \\
\hline Smáx2 /S & $\begin{array}{c}1.86 / 1.41=1.32<1.80 \\
\text { El dato SI es pertinente }\end{array}$ \\
\hline
\end{tabular}

No hay que realizar exclusiones ya que los valores extremos de los datos obtenidos de material que pasa el tamiz No. 200, cumplen con el criterio de Chauvenet, por lo cual se pude trabajar con la totalidad de los datos obtenidos de los ensayos de laboratorio.
Tabla 5. Módulo de Finura (MF).

\begin{tabular}{|c|c|}
\hline Media aritmética & $\mathrm{X}=2.63$ \\
\hline Desviación estándar & $\mathrm{S}=0.30$ \\
\hline Valor crítico de Chauvenet & $1.80($ PARA N=7) \\
\hline Smáx1 & $12.21-2.631=0.42$ \\
\hline Smáx1 /S & $\begin{array}{c}0.42 / 0.30=1.40<1.80 \\
\text { el dato SI es pertinente }\end{array}$ \\
\hline Smáx2 & $13.10-2.631=0.47$ \\
\hline Smáx2 /S & $\begin{array}{c}0.47 / 0.30=1.57<1.80 \\
\text { el dato SI es pertinente }\end{array}$ \\
\hline
\end{tabular}

No es necesario realizar exclusiones.

Tabla 6. Contenido de materia orgánica.

\begin{tabular}{|c|c|}
\hline Media aritmética & $\mathrm{X}=2.97$ \\
\hline Desviación estándar & $\mathrm{S}=0.37$ \\
\hline Valor crítico de Chauvenet & $1.80($ PARA N=7) \\
\hline Smáx1 & $12.44-2.971=0.53$ \\
\hline Smáx1 /S & $\begin{array}{c}0.42 / 0.37=1.43<1.80 \\
\text { el dato SI es pertinente }\end{array}$ \\
\hline Smáx2 & $13.11-2.971=0.14$ \\
\hline Smáx2 /S & $\begin{array}{c}0.14 / 0.37=0.38<1.80 \\
\text { el dato SI es pertinente }\end{array}$ \\
\hline
\end{tabular}

No es necesario realizar exclusiones.

Tabla 7. Humedad \%.

\begin{tabular}{|c|c|}
\hline Media aritmética & $\mathrm{X}=3.61$ \\
\hline Desviación estándar & $\mathrm{S}=0.38$ \\
\hline Valor crítico de Chauvenet & $1.80($ PARA N=7) \\
\hline Smáx1 & $13.21-3.611=0.40$ \\
\hline Smáx1 /S & $\begin{array}{c}0.40 / 0.38=1.05<1.80 \\
\text { el dato SI es pertinente }\end{array}$ \\
\hline Smáx2 & $14.12-3.611=0.51$ \\
\hline Smáx2 /S & $\begin{array}{c}0.51 / 0.38=1.34<1.80 \\
\text { el dato SI es pertinente }\end{array}$ \\
\hline
\end{tabular}

No es necesario realizar exclusiones.

Tabla 8. Absorción \%

\begin{tabular}{|c|c|}
\hline Media aritmética & $\mathrm{X}=1.72$ \\
\hline Desviación estándar & $\mathrm{S}=0.08$ \\
\hline Valor crítico de Chauvenet & $1.80(\mathrm{PARA} \mathrm{N}=7)$ \\
\hline Smáx1 & $11.63-1.721=0.09$ \\
\hline Smáx1 /S & $\begin{array}{c}0.09 / 0.08=1.13<1.80 \\
\text { el dato SI es pertinente }\end{array}$ \\
\hline Smáx2 & $11.85-1.721=0.13$ \\
\hline
\end{tabular}




\begin{tabular}{|c|c|}
\hline Smáx2 /S & $\begin{array}{c}0.13 / 0.08=1.63<1.80 \\
\text { el dato SI es pertinente }\end{array}$ \\
\hline
\end{tabular}

No es necesario realizar exclusiones.

\subsection{Resultados del análisis químico}

Resultados del análisis químico se encuentra Tabla 9 .

Tabla 9. Resultados estudio mineralógico barrido de las calles.

\begin{tabular}{|c|c|}
\hline DETERMINACIÓN ( $\%$ Masa ) & PROMEDIO \\
\hline Silicio expresado como $\mathrm{SiO} 2$ & 91,03 \\
\hline Aluminio total expresado como $\mathrm{A1} 2 \mathrm{O} 3$ & N.A \\
\hline Hierro total expresado $\mathrm{Fe} 2 \mathrm{O} 3$ & 1,17 \\
\hline Calcio expresado como $\mathrm{CaO}$ & 0,69 \\
\hline Magnesio expresado como $\mathrm{MgO}$ & 0,15 \\
\hline Sodio expresado como Na2O & $<0,1$ \\
\hline Potasio expresado como $\mathrm{K} 2 \mathrm{O}$ & 0,29 \\
\hline Manganeso expresado como $\mathrm{MnO} 2$ & 0,00 \\
\hline Titanio expresado TiO2 & 0,26 \\
\hline Fósforo expresado como $\mathrm{P} 2 \mathrm{O} 5$ & 0,10 \\
\hline Azufre expresado como $\mathrm{SO} 3$ & 0,040 \\
\hline Humedad a $105^{\circ} \mathrm{C}$ & 0,24 \\
\hline Pérdidas por calcinación $\mathrm{A} 1000^{\circ} \mathrm{C}$ & $\mathrm{NA}$ \\
\hline
\end{tabular}

Fuente:. Informe de Resultados de Análisis - I.D. Muestra 18755-E, Ingeominas.

\section{CONCLUSIONES}

Las cantidades de material obtenidas de barrido de las calles generadas por las diferentes concesiones de servicios y los diferentes tipos de recolección como se muestran en la Tabla 4, son significativas para tener en cuenta como una fuente de material más, Fig. 1, donde se puede ver que solo en el año 2009 se recogió la no despreciable cifra de $99,739.5$ toneladas.

Después de analizar 7 muestras extraídas de los 3 tipos de recolección y con base en los resultados obtenidos de este material, se pueden identificar las siguientes características del material obtenido del barrido de las calles en la localidad de Usaquén en Bogotá:

El análisis granulométrico generado mostrado en la Fig. 10 y Tabla 2 no presenta una tendencia aceptable dentro de los límites admitidos por la normatividad vigente como material de construcción en la preparación de mezclas de concreto, lo mismo que sucede con la mayoría de las muestras de arena natural, aunque teniendo estas granulometrías se pueden implementar métodos y formas para optimizar el material para usarlo como agregado en la preparación de mezclas.

El alto contenido de material Pasa 200 con un porcentaje promedio de $8.25 \%$ es nocivo y puede tener efectos perjudiciales para usarlo como material en la preparación de mezclas de concreto, aunque puede llegar a usarse realizando una optimización como materia en otros usos, como fabricación de mampuestos o elementos prefabricados de construcción, o como agregado fino en la preparación de morteros de pega para revestimientos.

Dentro de la clasificación de Suelos SUCS este material se encuentra dentro de los valores admitidos y determina que es un material bien gradado pero con presencia de limos (SW-SM) como se relaciona en la Tabla confirmando lo expuesto en el punto anterior por su alto contenido de Pasa No. 200.

Respecto al módulo de finura establecido, en promedio da como resultado 2,63, que determina la tendencia del aumento de este valor, es directamente proporcional a una gradación más gruesa, están entre los valores de 2,15 y 3,38 ; con esto se determina aceptable y se ubica dentro del rango de las arenas lo que permite establecer que si es utilizado en la preparación de mezclas de concreto o mortero su manejabilidad es buena.

El ensayo colorimétrico muestra un resultado promedio de 2,97 (Tabla 2); teniendo en cuenta este valor tan cercano al límite que corresponde a 3 , es permisible en cuanto al contenido de materia orgánica, aunque como lo demuestran varios autores el color establecido no siempre por ser un color oscuro corresponde a una sustancia perjudicial para ser utilizado como material de construcción [13].

En la parte de la composición química presenta un $91,03 \%$ de Silicio según mineralogía realizada, esto da un punto más a la hipótesis presentada al compararlo con una arena ya que el mayor componente es el dióxido de silicio, aunque al comparar 


\section{con-ciencias}

las Tablas 4 y 5 se pueden establecer porcentajes mayores a los de una arena convencional y un menor porcentaje para el componente de Sílice.

Es fundamental mencionar acerca de la absorción que tienen individualmente y en conjunto las muestras. Los valores obtenidos mediante esta práctica sobre la absorción permiten concluir y aceptar la cantidad de agua que pude absorber, estas cantidades indican que las muestras llegan en un estado de (SSS) saturado y superficialmente seco. Es decir, que estas al tacto no presentan humedad pero sus poros contienen agua. En el caso del agregado fino el promedio de absorción es de $1.72 \%$.

Finalmente, se procede a determinar el peso unitario por medio de dos prácticas; peso unitario suelto y peso unitario compactado. Los criterios que se toman en cuenta para este ensayo son los siguientes:

Los dos métodos para determinar el peso unitario según la norma NTC-92 son aceptables, permitiendo concluir y admitir con respecto al error un valor positivo, esto es aceptable, debido a que, los ensayos son realizados por la misma persona.

En un aspecto económico, después de obtener la densidad como está relacionado en la Tabla 2, se establece el valor del $\mathrm{m}^{3}$ de material obtenido del barrido de las calles, al tener la densidad aparente de $2,68 \mathrm{Ton} / \mathrm{m}^{3}$ y teniendo en cuenta que su recolección por $\mathrm{km}$ es de $53,74 \mathrm{Kg}=0,0537 \mathrm{Ton}$. Además que el $\mathrm{km}$ de barrido promedio según es de $\$$ 17.398 [1] pesos se puede determinar lo siguiente:

Por km se recoge 0,02 m3 de material obtenido del barrido de las calles, para un $\mathrm{m} 3$ se tendrían que barrer $50 \mathrm{~km}$., con lo cual para el Estado el m3 de material obtenido de las calles cuesta $\$ 869.900$ pesos, un costo que se puede minimizar en su disposición final al establecer un aprovechamiento de este recurso como material de construcción.

\section{REFERENCIAS}

[1] UAESP - Unidad Administrativa Especial de Servicios Públicos, Información material estadístico e información general del barrido, 2009EE 9128, 29 de octubre de 2009.

[2] M. Solano. Las basuras en Bogotá de la Colonia al siglo XXI, Bogota, 2010, [en línea], Disponible en http://www.bogota. gov.co/ portel/librería/php/x frame detalle.php?id $=40680$

[3] Instituto Distrital de Cultura y Turismo, Alcaldía Mayor de Bogotá D.C, Ficha Técnica Turística Localidad de Usaquén, Bogotá D.C.: IDCT, 2004.

[4] R. Cabana, Clasificación de materiales, [en línea] Disponible en: http:/www. monografias.com/trabajos6/clame/clame. shtml.

[5] LIME S.A. E.S.P. Sexta audiencia de rendición de cuentas. Contrato 054 -08 suscrito con la Alcaldía Mayor de Bogotá, 2009.

[6] M. Jaeger, Nagel, R. Sidney and R. Behringer, "Granular Solids, Liquids, and Gases", Reviews of Modern Physics, 1996.
[7] M. Colomer, J. Francisco, A. Gallardo, Tratamiento y gestión de residuos sólidos, Valencia: UPV, 2007.

[8] CEAC, Enciclopedia del Encargado de Obras, Materiales para la Construcción, CEAC S.A: Barcelona, cuarta Edición, 1983.

[9] E. RIVA, Naturaleza y materiales del concreto, Perú: ACI, 2011.

[10] M. Braja, Principios de ingeniería de cimentaciones, cuarta edición, México: Internacional Editores Thompson, 2006.

[11] A. Cadena, "Aspectos Técnicos en el manejo de los residuos sólidos", Hacia un Pacto Limpio, Reunión sobre manejo de residuos sólidos y reciclaje, Bogotá: Ministerio de Medio Ambiente, 1995.

[12] A. García, "Propiedades físicas de los residuos sólidos urbanos". Libro verde de medio ambiente urbano, 2007.

[13] Enciclopedia Virtual, Características químicas de los residuos sólidos urbanos, [en línea], Disponible en: http:// www.ambientum.com/enciclopedia/ residuo/1.26.31.11r.html. 